\title{
Anteil und Wassergehalt des Splints von schweizerischen Fichten und Tannen unterschiedlicher Vitalität*
}

\author{
J. Sell, G. R. Schnell und M. Arnold \\ Abteilung Holz der EMPA Dübendorf, Schweiz
}

\begin{abstract}
Je zehn vitale und geschädigte Fichten und Tannen wurden in den Wintern 1984/85 und 1985/86 zu vergleichenden Untersuchungen auf vier Schweizer Standorten gefällt. Messungen der Breite und des Wassergehalts des Splintholzes und daraus abgeleiteter Splintmerkmale im Erdstamm- und Wipfelbereich führten zu folgenden Ergebnissen: Der Splintanteil der kranken Bäume war erheblich geringer als der gesunder Vergleichsbäume. Das Defizit der durchschnittlichen absoluten Splintbreite betrug bei kranken Fichten rd. 30\% im unteren Stammabschnitt und $40 \%$ im Wipfelbereich; die entsprechenden Werte bei Weißtannen sind rd. $40 \%$ und $20 \%$. Bei den kranken Fichten waren die Splintanteile auf dem alpinen und voralpinen Standort besonders gering, auf dem Mittelland-Standort am wenigsten vermindert. Kranke Tannen wiesen auf allen Standorten im unteren Stammbereich einen etwa gleich verminderten Splintanteil auf; im Wipfelbereich war die Verminderung auf dem voralpinen Standort signifikant größer als anderswo. Der Wassergehalt des Splints gesunder und kranker Fichten und Tannen war meist nicht signifikant verschieden. Die abgeleitete Größe „Wasserhaushalt“ führt zu ähnlichen Aussagen wie die Einzelmeßgrößen.
\end{abstract}

Portion and water content of the sapwood of Swiss spruce and white fir of various vitality

For measuring sapwood data, ten healthy and ten diseased trees of spruce and white fir were cut in winter 1984/85 and 1985/86 at four different Swiss locations. The portion of sapwood of the diseased trees turned out to be considerably less than for healthy trees. The deficit in average sapwood width of diseased trees was $30 \%$ (trunk) to $40 \%$ (crown) for spruce and $40 \%$ to $20 \%$ for white fir. The sapwood portion of diseased spruce was particularly low at alpine and pre-alpine locations. The sapwood reduction of diseased white fir was similar at all locations, except for the crowns of pre-alpine trees which showed a significantly greater sapwood reduction. The sapwood water content of both diseased and healthy trees of both species exhibited mostly non-significant differences. Derived terms like "water budget" provided similar information as the original data.

\section{Einleitung}

Im Rahmen einer Erhebung über die Holzqualität gesunder und kranker Fichten und Tannen wurden Kenngrößen des Splintholzes ermittelt. Ziel der Arbeit war nicht, die Ursachen unterschiedlicher Splintmerkmale klären zu helfen, sondern solche Unterschiede quantitativ darzustellen.

\footnotetext{
* Untersuchungen im Rahmen des Programms Sanasilva, Teilprojekt „Holzqualität". Kurzfassung des EMPA-Forschungs- und Arbeitsberichts Nr. 115/15 (Schnell et al. 1987)
}

\section{Material und Methode}

Das Probenmaterial, insgesamt 162 Bäume, entstammt Fichten/Tannenbeständen aus den Waldungen der Gemeinden Lenz und Malans in Graubünden (alpine Standorte), Bilten in Glarus (voralpin), Buchs-Rohr-Suhr im Aargau (Mittelland) und Lausanne in der Waadt (Jorat-Hügelland). Pro Standort standen Stammabschnitte von je 10 gesunden und kranken Fichten und Tannen zur Verfügung, von denen jeweils ein Abschnitt von $80 \mathrm{~cm}$ Länge und eine Stammscheibe von $10 \mathrm{~cm}$ Dicke aus dem unteren Stammabschnitt (Brusthöhe bis $5 \mathrm{~m}$ Stammhöhe) sowie eine Scheibe von $10 \mathrm{~cm}$ Dicke aus dem Wipfelbereich untersucht wurden. Die Entnahme erfolgte an frisch gefällten Bäumen vom Dezember 1984 bis Februar 1985 und vom November 1985 bis Februar 1986. Weitere Charakteristika der Standorte und der untersuchten Bäume sind in Tabelle 1 zusammengestellt.

Die Bestimmung des Wassergehalts des waldfrischen Holzes machte die umgehende Verarbeitung der Stammscheiben erforderlich und erfolgte jeweils an 4 Querschnittstreifen; diese Streifen entsprachen 4 senkrecht zueinander

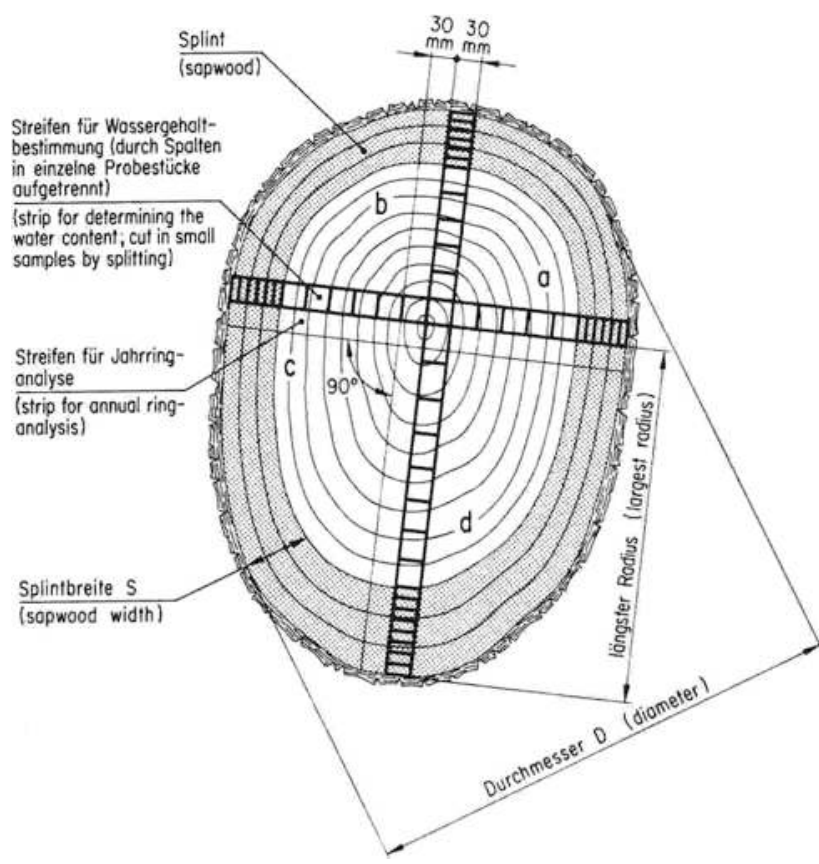

Bild 1. Entnahmeschema für die vier radialen Probestreifen zur Bestimmung des Wassergehaltes im Darrverfahren

Fig. 1. Scheme of split-sampling for determining the water content by the oven-dry method 
Tabelle 1. Bestandesbeschreibung und Kenndaten der Probebäume

\begin{tabular}{|c|c|c|c|c|c|}
\hline & \multicolumn{2}{|l|}{ Alpen } & \multirow{2}{*}{$\begin{array}{l}\text { Voralpen } \\
\text { Ta und Fi }\end{array}$} & \multirow{2}{*}{$\begin{array}{l}\text { Mittelland } \\
\text { Ta und Fi }\end{array}$} & \multirow{2}{*}{$\begin{array}{l}\text { Jorat } \\
\text { (Hügelland) } \\
\text { Ta und } \mathrm{Fi}\end{array}$} \\
\hline & $\mathrm{Ta}$ & $\mathrm{Fi}$ & & & \\
\hline Standort & Malans/GR & Lenz/GR & Bilten/GL & Buchs-Rohr-Suhr/AG & Lausanne/VD \\
\hline Höhe ü. M., m & 810 bis 1000 & 1430 & 1200 & 370 bis 390 & 740 bis 780 \\
\hline Exposition & SW & W & NW & $\mathrm{W}$ bis $\mathrm{NW}$ & SSO \\
\hline Topographie & Hang & Ebene & $\begin{array}{l}\text { Kuppe, Mulde } \\
\text { Hang, Ebene }\end{array}$ & Ebene & Ebene \\
\hline Geologie & $\begin{array}{l}\text { kalkreicher } \\
\text { Bündnerschiefer }\end{array}$ & Schotter & Nagelfluh & $\begin{array}{l}\text { Niederterassen- } \\
\text { Schotter }\end{array}$ & Molasse \\
\hline Boden & $\begin{array}{l}\text { tiefgründige } \\
\text { Renzina }\end{array}$ & $\begin{array}{l}\text { nachgründige } \\
\text { Renzina }\end{array}$ & $\begin{array}{l}\text { flach bis tief- } \\
\text { gründiger Mergel }\end{array}$ & $\begin{array}{l}\text { mittelgründige } \\
\text { kalkarme } \\
\text { Braunerde }\end{array}$ & $\begin{array}{l}\text { mitteigründige } \\
\text { leicht podsolige } \\
\text { saure Braunerde }\end{array}$ \\
\hline Niederschlag, $\mathrm{mm} / \mathrm{Jahr}$ & 1066 & 1133 & 1834 & 1061 & 1084 \\
\hline $\begin{array}{l}\text { Durchschnitts- } \\
\text { temperatur, "C/Jahr }\end{array}$ & 4.9 & 4.9 & 6.7 & 8.3 & 9.6 \\
\hline \multicolumn{6}{|l|}{$\begin{array}{l}\text { Bestandes- } \\
\text { charakteristika }\end{array}$} \\
\hline Betriebsform & Hochwald & Mittelwald & Hochwald & Hochwald & Hochwald \\
\hline Entwicklungsstufe & Altholz & $\begin{array}{l}\text { BH II } \\
\text { Altholz }\end{array}$ & Altholz & $\begin{array}{l}\text { BH II, } \\
\text { Altholz }\end{array}$ & $\begin{array}{r}\text { BH I + II } \\
\text { Altholz }\end{array}$ \\
\hline $\begin{array}{l}\text { Baumarten- } \\
\text { Mischung }\end{array}$ & $\begin{array}{l}96 \% \mathrm{Ta} \\
5 \% \mathrm{Bu}, \mathrm{BAh}\end{array}$ & $100 \%$ Fichte & $50 \% \mathrm{Fi}, 50 \% \mathrm{Ta}$ & $\begin{array}{l}90 \% \mathrm{Fi}, 5 \% \text { Ta } \\
5 \% \mathrm{Bu}, \mathrm{BAh}, \mathrm{Es}\end{array}$ & $50 \% \mathrm{Fi}, 50 \% \mathrm{Ta}$ \\
\hline \multicolumn{6}{|l|}{ Probebäume } \\
\hline Fichte, gesund & & 140 bis 195 & 115 bis 200 & 70 bis 75 & 80 bis 100 \\
\hline Fichte, krank & & 135 bis 205 & 90 bis 170 & 75 bis 125 & 75 bis 100 \\
\hline Tanne, gesund & 50 bis 80 & & 100 bis 175 & 80 bis 100 & 75 bis 180 \\
\hline Tanne, krank & 55 bis 125 & & 110 bis 140 & 75 bis 100 & 85 bis 105 \\
\hline \multicolumn{6}{|l|}{ Höhe, m } \\
\hline Fichte, gesund & & 20 bis 26 & 20 bis 33 & 29 bis 38 & 29 bis 36 \\
\hline Fichte, krank & & 16 bis 24 & 21 bis 26 & 31 bis 40 & 23 bis 34 \\
\hline Tanne, gesund & 25 bis 32 & & 24 bis 31 & 21 bis 38 & 17 bis 40 \\
\hline Tanne, krank & 23 bis 34 & & 12 bis 27 & 27 bis 40 & 24 bis 34 \\
\hline \multicolumn{6}{|l|}{$\begin{array}{l}\text { Brusthöhen- } \\
\text { durchmesser, cm }\end{array}$} \\
\hline Fichte, gesund & & 37 bis 50 & 29 bis 83 & 40 bis 55 & 34 bis 61 \\
\hline Fichte, krank & & 31 bis 44 & 29 bis 96 & 40 bis 62 & 27 bis 59 \\
\hline Tanne, gesund & 43 bis 66 & & 44 bis 61 & 37 bis 58 & 34 bis 75 \\
\hline Tanne, krank & 30 bis 74 & & 29 bis 77 & 42 bis 62 & 35 bis 65 \\
\hline \multicolumn{6}{|l|}{ Kronenanteil. \% } \\
\hline Fichte, gesund & & 61 bis 90 & 30 bis 60 & 47 bis 75 & 31 bis 58 \\
\hline Fichte, krank & & 45 bis 87 & 37 bis 73 & 46 bis 72 & 25 bis 66 \\
\hline Tanne, gesund & 30 bis 84 & & 30 bis 58 & 52 bis 73 & 40 bis 94 \\
\hline Tanne, krank & 22 bis 69 & & 9 bis 83 & 59 bis 81 & 16 bis 83 \\
\hline \multicolumn{6}{|l|}{ Probeentnahme } \\
\hline $\begin{array}{l}\text { Höhe im unteren } \\
\text { Stammbereich, m }\end{array}$ & 5.0 & 1.0 bis 2.0 & 1.0 bis 4.0 & 1.0 & 1.0 \\
\hline
\end{tabular}

BAh Bergahorn, Bu Buche, Es Esche, Fi Fichte, Ta Wcisstanne

stehenden Stammradien, wobei im Falle von unrunden Stämmen jeweils der längste Radius miteinbezogen wurde (Bild 1). Auf diese Weise konnte die Exzentrizität des Stammes bei der Bestimmung des Wassergehalts berücksichtigt werden.

Die 4 radialen Streifen wurden scheibenweise gespalten, im Splintbereich in 2 bis $5 \mathrm{~mm}$ dicke, im Kernbereich in 10 bis $20 \mathrm{~mm}$ dicke Probestücke, an denen umgehend der Wassergehalt nach DIN 52183 (Prüfung von Holz: Bestimmung des Feuchtigkeitsgehaltes) bestimmt wurde. Aus der Verteilung des Wassergehalts über dem Stammradius kann die Splintbreite und die kreisäquivalente oder ellipsenäquivalente Splintfläche berechnet werden. Dabei ist von Bedeutung, wo die Splint/Kerngrenze angenommen wird; aufgrund verschiedener Indizien haben wir den Bereich mit Wassergehalten $\geqq 100 \%$ als Splint betrachtet.
Die Distanz vom Kambium bis zur Splint/Kerngrenze wird ins Verhältnis zum gesamten Stammradius gesetzt und mit ,relative Splintbreite“ (in Prozenten) bezeichnet; aus den gleichen Daten wird die rel. Splintfläche berechnet. Eine weniger aufwendige Methode zur Bestimmung der Splintfläche ist die bildanalytische Auswertung von Fotografien (Farbdiapositive) von Stammquerschnitten (Arnold et al. 1986).

Um die Wasserversorgung des Splints noch weitergehend zu charakterisieren, schien die multiplikative Verknüpfung von Wassergehalt und Splintanteil sinnvoll. Dieses Produkt, das man mit "Wasserhaushalt" bezeichnen kann, läßt sich mit geringem Datenaufwand berechnen. Vor allem können hierzu auch die mit dem Bildanalysator ermittelten rel. Splintflächen eingesetzt werden; ferner genügt es, den Wassergehalt an einem vom Kern abgetrennten Splintabschnitt (unmittelbar nach der Fällung) zu bestimmen. 


$$
\text { Nr. } 17 \text { Fichte-Wipfel Nr. } 01
$$

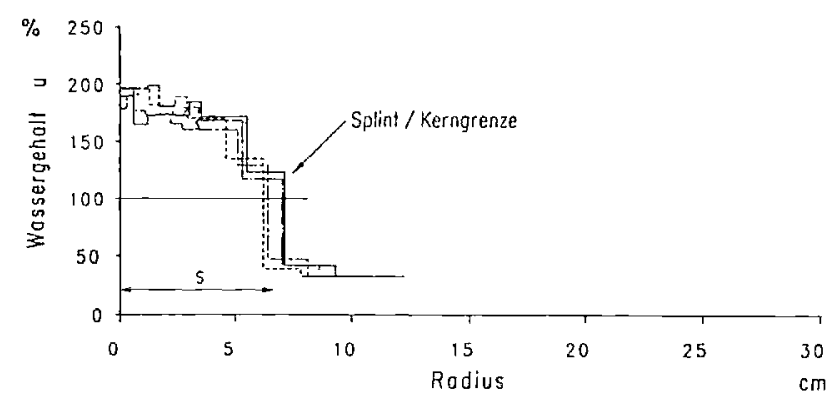

Nr. 17

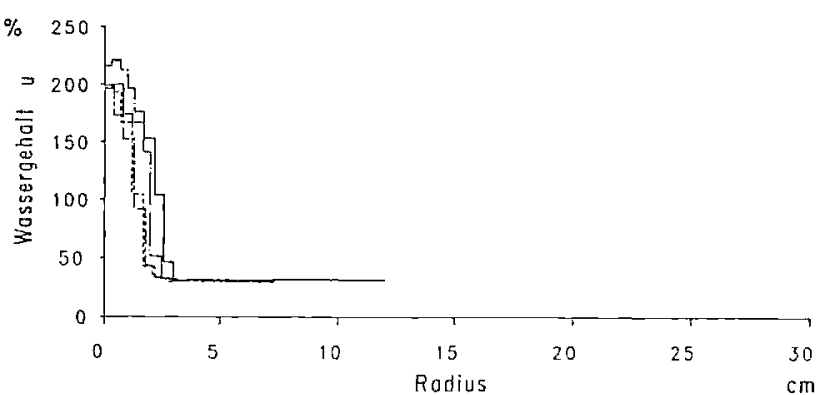

Nr. 01
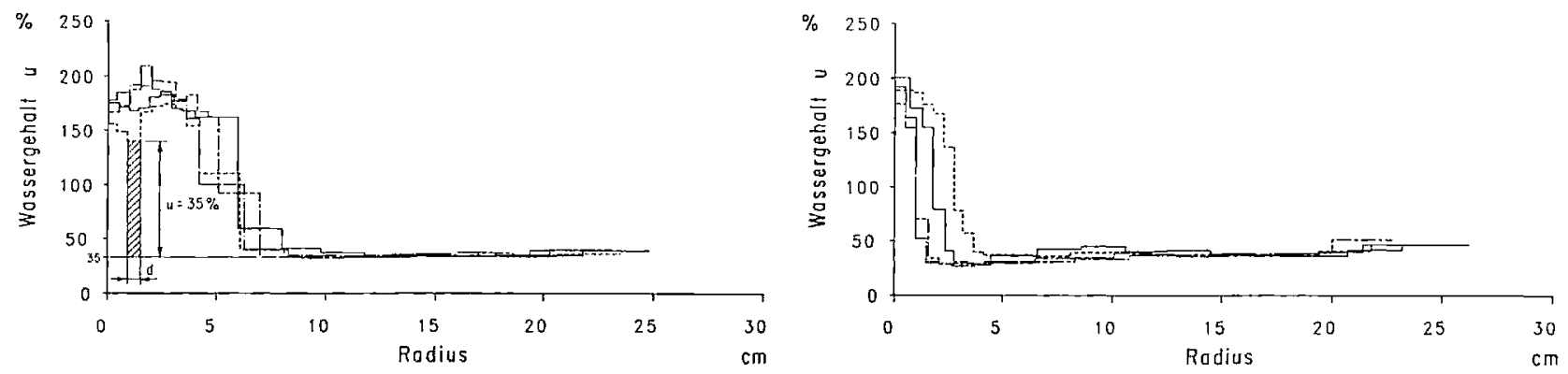

gesund

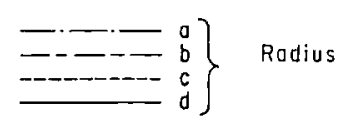

krank

Bild 2. Wassergehaltsverteilung über dem Stammradius ausgewähiler gesunder bzw. kranker Fichten

Fig. 2. Distribution of water content over the stem radius of single healthy and diseased spruces

\section{Ergebnisse und Diskussion}

Bereits ein erster visueller Eindruck von den Verteilungen des Wassergehalts über den Stammradius und insbesondere von den Splintbreiten der verschiedenen Probenkollektive zeigt, daß zwischen der von Auge beurteilten Vitalität (Kronenansprache) der Bäume und den Splintdaten ein Zusammenhang besteht (Bild 2). Ganz generell läßt sich feststellen, daß vor allem der Anteil und teilweise auch der Wassergehalt des Splints bei wenig vitalen Fichten und Tannen wesentlich geringer sind als bei ausgesprochen vitalen Bäumen.

Die Verteilungen des Wassergehalts im Splint von Fichte und Tanne weisen die erwarteten Unterschiede auf, wobei insbesondere der häufig nasse Kern der Tanne ins Auge fällt. Innerhalb der Baumkollektive bestehen naturgemäß Unterschiede des Wassergehalts im unteren Stammbereich und im Wipfel (Tabelle 2).

\subsection{Wassergehalt des Splints}

Wie in Abschnitt 2 erläutert, wurde der Grenz-Wassergehalt zwischen Splint und Kern in der vorliegenden Untersuchung mit $\mathrm{u} \geqq 100 \%$ definiert. Bei der schrittweisen Wassergehaltsbestimmung entlang von Stammradien hatte sich bei der Fichte und bei der Tanne (ohne Naßkern) gezeigt, daß der Abfall des Wassergehalts auf der Splint/Kerngrenze ziemlich steil verläuft (meist innerhalb weniger Millimeter radialer Distanz) und durch diesen Grenzwert gut charakterisiert ist (Bild 2). (Auf frischen Stammquerschnitten lag die Grenze zwischen dem nassen und darum dunkleren Splint und dem trocknen, hellen Kern offenbar ebenfalls im Bereich eines Wassergehalts von $100 \%$.)

Dieser Befund darf allerdings nicht verallgemeinert werden. Vielmehr steht aufgrund umfangreicher äIterer Untersuchungen fest, daß der Wassergehalt, vor allem im kernna-

Tabelle 2. Über alle Standorte gemittelte Breiten und Wassergehalte des Splints gesunder und kranker Fichten und Tannen. Die in Klammern stehenden Werte geben die jeweilige Meßgröße der kranken Bäume im Vergleich zu den Werten gesunder Bäume an.

\begin{tabular}{|c|c|c|c|c|c|c|c|c|c|c|}
\hline \multirow{3}{*}{\multicolumn{2}{|c|}{ Kollektive }} & \multirow[b]{4}{*}{$n$} & \multicolumn{8}{|l|}{ Meßgrößen } \\
\hline & & & \multirow{2}{*}{\multicolumn{2}{|c|}{$\begin{array}{l}\text { Probenscheiben- } \\
\text { durchmesser } \\
D \mathrm{~cm}\end{array}$}} & \multirow{2}{*}{\multicolumn{2}{|c|}{$\begin{array}{l}\text { Wassergehalt } \\
\text { im Splint } \\
\bar{u} \%\end{array}$}} & \multicolumn{4}{|c|}{ Splintbreite (Darrverfahren) } \\
\hline & & & & & & & \multicolumn{2}{|c|}{ Absolut $S_{\mathrm{abs}} \mathrm{cm}$} & \multicolumn{2}{|c|}{ Relativ $S_{\mathrm{rel}} \%$} \\
\hline Baumart & Vitalität & & Erdstamm & Wipfel & Erdstamm & Wipfel & Erdstamm & Wipfel & Erdstamm & Wipfel \\
\hline Fichte & $\begin{array}{l}\text { Gesund } \\
\text { Krank }\end{array}$ & $\begin{array}{l}43 \\
38\end{array}$ & $\begin{array}{l}42.6 \\
37.1(87 \%)\end{array}$ & $\begin{array}{l}19.3 \\
18.9(98 \%)\end{array}$ & $\begin{array}{l}164 \\
152(93 \%)\end{array}$ & $\begin{array}{l}161 \\
152(94 \%)\end{array}$ & $\begin{array}{l}4.4 \\
3.0(68 \%)\end{array}$ & $\begin{array}{l}4.1 \\
2.6(63 \%)\end{array}$ & $\begin{array}{l}20.6 \\
15.7(76 \%)\end{array}$ & $\begin{array}{l}41.3 \\
27.1(66 \%)\end{array}$ \\
\hline Tanne & $\begin{array}{l}\text { Gesund } \\
\text { Krank }\end{array}$ & $\begin{array}{l}40 \\
40\end{array}$ & $\begin{array}{l}46.1 \\
40.3(87 \%)\end{array}$ & $\begin{array}{l}21.5 \\
21.1(98 \%)\end{array}$ & $\begin{array}{l}152 \\
142(93 \%)\end{array}$ & $\begin{array}{l}168 \\
161(96 \%)\end{array}$ & $\begin{array}{l}6.9 \\
4.1(59 \%)\end{array}$ & $\begin{array}{l}5.5 \\
4.4(80 \%)\end{array}$ & $\begin{array}{l}29.8 \\
20.6(69 \%)\end{array}$ & $\begin{array}{l}52.5 \\
41.8(80 \%)\end{array}$ \\
\hline
\end{tabular}


hen Splint der Nadelhölzer, in starkem Maße von der Jahreszeit (in geringerem Maße von der Tageszeit) bzw. vom Klima, vom Standort und auch von anderen Faktoren abhängt (Burmester 1980; Huber 1956; Langner 1932; u. a.). Auch im Rahmen von neueren vergleichenden Untersuchungen des Holzes geschädigter und gesunder Fichten wurden andere mittlere Wassergehalte als bei unseren Bäumen gemessen (Frühwald et al. 1984; Rademacher et al. 1986): In diesen Untersuchungen wurden an sehr kranken Fichten (Schadklasse 3) im inneren Splintbereich ein - im Vergleich zu gesunden Bäumen - um rund ein Drittel bis zur Hälfte reduzierter Wassergehalt festgestellt; dadurch lag auch der mittlere Splint-Wassergehalt kranker Bäume deutlich tiefer als bei gesunden Bäumen. Demgegenüber betrug die mittlere Differenz der Wassergehalte des Splints bei den von uns untersuchten Bäumen weniger als ein Zehntel, mit einer Schwankungsbreite zwischen 2 und 15\%. Für diese abweichenden Befunde mögen unterschiedliche Standort-Charakteristika und Fälldaten verantwortlich sein, vermutlich aber auch die Tatsache, daß ein erheblicher Teil der von den genannten Autoren untersuchten Bäume unmittelbar nach einem extrem trocknen Sommer im Winter 1983/84 gefällt worden waren, unsere Bäume hingegen im Winter 1984/85 und 1985/86, als sich die Wasserversorgung des Stammes nach Perioden mit normalem oder sogar hohem Niederschlag - wieder stabilisiert hatte.

Vergleicht man die gemittelten Wassergehalte des Splintholzes der Bäume aus den vier Schweizer Standorten, fallen insgesamt die bemerkenswert geringen Unterschiede auf. So liegt der Wassergehalt in relativ engen Grenzen (jeweils unterer Stammabschnitt). Er beträgt bei

gesunden Fichten: $154 \% \leqq u \leqq 176 \%$ (wobei der kleinste Wert im Mittelland und der größte beim Jorat-Hügelstandort gefunden wurde);

kranken Fichten: $150 \% \leqq u \leqq 156 \%$ (Alpen bzw. Voralpen); gesunden Tannen: $141 \% \leqq u \leqq 164 \%$ (Jorat bzw. Alpen);

kranken Tanne: $135 \% \leqq u \leqq 151 \%$ (Mittelland bzw. Voralpen).

Der Splint im Wipfelbereich der Fichten wies an den vier Standorten sehr ähnliche Wassergehalte wie im unteren Stammabschnitt auf, während der Wassergehalt im Splint des Weißtannenwipfels bei allen Standorten um rd. 10 bis $20 \%$ höher lag als der Splint im unteren Stammabschnitt.

Die Streuung der Wassergehalte, innerhalb der einzelnen Kollektive war überwiegend nicht sehr groß. Die zumeist ge* ringen Unterschiede der Wassergehalte zwischen den gesunden und den kranken Bäumen sind bei den meisten Standorten statistisch nicht gesichert (Signifikanztest mit dem verteilungsunabhängigen Wilcoxon-U-Test).

\subsection{Splintbreite, Splintfläche}

Die gemäß dem Schema von Bild 1 an 4 Stammradien bestimmte Splintbreite wurde pro Baum und sodann über die Bäume jedes Kollektivs (meist 10 Exemplare) gemittelt. Auf allen 4 Standorten ist diese mittlere absolute Splintbreite $S$ - und ebenso die rel. Splintfläche $A_{\mathrm{D} \text {,rel }}$ - sowohl im unteren Stammabschnitt wie im Wipfelbereich bei den kranken Fichten und Tannen geringer als bei den gesunden Vergleichsbäumen. So beträgt der Bereich der Splintbreite und rel. Splintfläche im unteren Stammabschnitt bei

gesunden Fichten: $3,6 \mathrm{~cm} \leqq S \leqq 4,9 \mathrm{~cm}$ (Alpen- bzw. JoratHügellang) bzw. $33 \% \leqq A_{\mathrm{D}, \text { rel }} \leqq 40 \%$ (Voralpen bzw. Mittelland);

kranken Fichten: $2,1 \mathrm{~cm} \leqq S \leqq 4,4 \mathrm{~cm}$ (Alpen- bzw. Mittelland) bzw. $23 \% \leqq A_{\text {D.rel }} \leqq 35 \%$ (Voralpen und Mittelland).
Diese Unterschiede des Splintanteils gesunder und kranker Bäume sind statistisch gesichert, ausgenommen bei den Mittelland-Fichten, wo kein Unterschied der absoluten Splintbreite besteht. Im Wipfelbereich der Fichten sind die Unterschiede der Splintbreiten gesunder und kranker Bäume noch deutlicher (nun auch bei den Mittelland-Fichten) als beim Erdstamm.

Die Splintbreiten und rel. Splintflächen der gesunden ebenso wie der kranken Weißtannen waren auf allen Standorten deutlich und zumeist hoch gesichert größer als bei den entsprechenden Fichtenkollektiven; sie betrugen im unteren Stammabschnitt bei

gesunden Tannen: $6,1 \mathrm{~cm} \leqq S \leqq 7,9 \mathrm{~cm}$ (Mittelland bzw. Jorat-Hügelland) bzw. $46 \% \leqq A_{\mathrm{D}, \text { rel }} \leqq 58 \%$ (Mittelland bzw. Jorat-Hügelland);

kranken Tannen: $3,5 \mathrm{~cm} \leqq S \leqq 5,2 \mathrm{~cm}$ (Alpen bzw. Jorat-Hügelland) bzw. $27 \% \leqq A_{\mathrm{D}, \text { rel }} \leqq 43 \%$ (Mittelland bzw. JoratHügelland).

Weniger unterschiedlich sind indessen die Splintbreiten im Wipfelbereich der gesunden und kranken Tannen; diese Unterschiede sind teilweise nicht gesichert (Mittelland und Jorat).

Zusammenfassend ist festzustellen, daß das - gegenüber den gesunden Vergleichsbäumen - größte Defizit des für den Wasserhaushalt maßgebenden Splintanteils der kranken Fichten auf dem alpinen und auch auf dem voralpinen Standort ermittelt wurde: ca. 40 bis $50 \%$ geringere absolute Splintbreiten und ca. 30 bis $40 \%$ kleinere relative Splintflächen; dies gilı für den unteren Stammabschnitt ebenso wie für den Wipfelbereich. Am geringsten war das Defizit des Splintanteils bei den kranken Fichten vom MittellandStandort: 20 bis $25 \% \mathrm{im}$ Wipfelbereich und nur 0 bis $10 \%$ im unteren Stammabschnitt. Die kranken Tannen hatten im unteren Stammabschnitt gegenüber den gesunden Vergleichsbäumen auf allen Standorten rd. 35 bis $45 \%$ geringere absolute Splintbreiten und um 20 bis $40 \%$ geringere rel. Splintflächen; im Wipfelbereich betrug das Defizit der Splintbreite 15 bis $30 \%$ und das der rel. Splintfläche knapp 10 bis $20 \%$. Auch bei der Tanne traten die größten Defizite auf dem Alpen- und dem Voralpenstandort auf.

\subsection{Verknüpte Splintdaten}

Im Rahmen der vorliegenden Untersuchung erbrachte die Größe „Wasserhaushalt" keine weitergehende Differenzierung zwischen gesunden und kranken Fichten und Tannen, im Vergleich zur Splintbreite oder Splintfläche. Die Ursache dafür liegt darin, daß der Wassergehalt des Splints der von uns untersuchten kranken Bäume nicht signifikant niedriger lag als bei den gesunden Vergleichsbäumen und darum das Produkt Splintanteil $\times$ Wassergehalt durchschnittlich nicht oder nur unwesentlich beeinflußte. Dies ist anders, wenn kranke Bäume auch ein ausgeprägtes Wassergehalts-Defizit aufweisen, wie es z. B. von Frühwald et al. (1984) und Rademacher et al. (1986) an 1982/83 und 1984 gefällten Bäumen von verschiedenen deutschen Standorten festgestellt worden ist. In diesen deutschen Untersuchungen hatten die kranken Bäume nicht nur eine um rd. 20 bis $45 \%$ geringere Splintbreite, sondern auch einen wesentlich geringeren Splint-Wassergehalt (insbesondere im kernnahen Splintbereich). Daraus errechnet sich für mehrere der untersuchten deutschen Standorte ein um mehr als 50\% reduzierter Wasserhaushalt der kranken gegenüber den gesunden Fichten; demgegenüber betrug das Defizit des Wasserhaushalts der 1984/85 und 1985/86 gefällten Fichten von den 4 Schweizer Standorten 

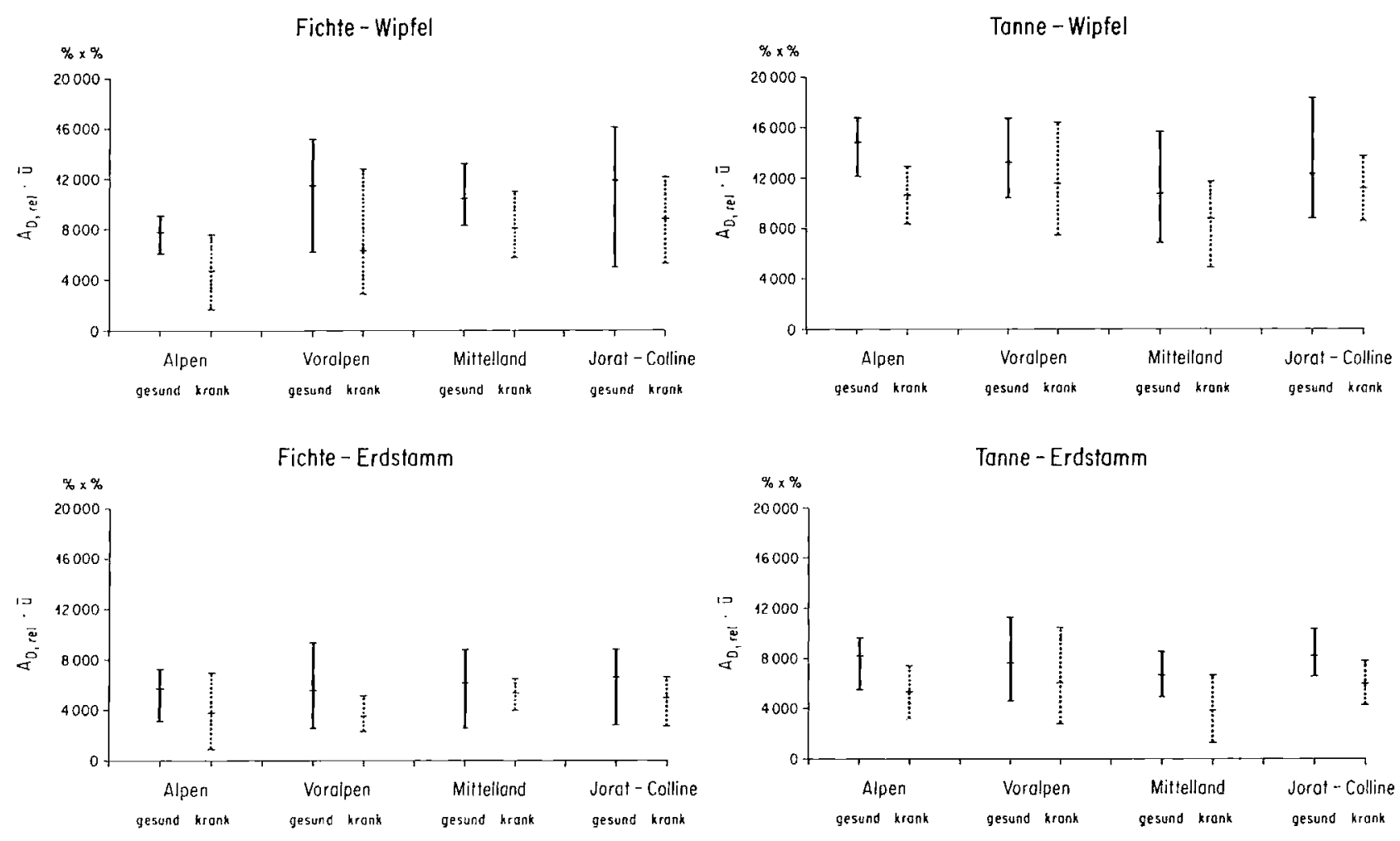

Bild 3. Abgeleitete Meßgröße „Wasserhaushalt" (Splintanteil $\times$ Wassergehalt) aller Probenkollektive; grafische Darstellung der Mittel- und Extremwerte

Fig. 3. Values of the derived term "water-budgel" (sapwood portion $\times$ water content) of all groups of samples; averages and extreme values

durchschnittlich nur rd. 25\% (unterer Stammabschnitt) und $35 \%$ (Wipfel).

Es ist im übrigen darauf hinzuweisen, daß die Werte des Wasserhaushalts von verschiedenen Standorten und Fälldaten nicht ohne weiteres miteinander verglichen werden können, weil sie von zahlreichen, zumeist unbekannten Faktoren komplex beeinflußt und stark zeitabhängig sind. Als Vergleichsgröße kranker und gesunder Bäume eines oder mehrerer Standorte ist der Wasserhaushalt aber geeigneter als die Splintbreite, weil er zugleich über Splintanteil und SplintWassergehalt informiert und, wie die zitierten deutschen Untersuchungen zeigen, als diagnostisches Hilfsmittel trennschärfer ist als die Einzelmeßgrößen. Auf diesen Sachverhalt stützt sich wohl auch der Vorschlag von Kučera (1986), am stehenden Baum mit Hilfe verschiedener MeBmethoden Splintdaten zu ermitteln.

\section{Zusammenhänge zwischen Splintdaten und Holzeigenschaften}

Das von der Holzabteilung der EMPA bearbeitete Teilprojekt "Holzqualität“ der Erhebung Sanasilva über den Gesundheitszustand des Schweizer Waldes befaßt sich mit der Untersuchung folgender Holzeigenschaften an den gleichen Bäumen, die auch der vorliegenden Studie zugrunde liegen: Gefügemerkmale (Jahrringbreite, Zellwanddicken), feuchtephysikalische Eigenschaften (Sorption, Schwind- und Quellmasse), mechanisch-technologische Eigenschaften (Biegefestigkeit, Biege-E-Modul, Bruchschlagarbeit, Druckscherfestigkeit), anwendungstechnische Eigenschaften (Trocknungsverhalten, Verleimbarkeit, Oberflächenbehandlung u. a.). Durch die Abteilung Biologie der EMPA werden auBerdem holzpathologische Aspekte und die Imprägnierbarkeit von Holz gesunder und kranker Bäume untersucht. Die
Ergebnisse dieser umfangreichen Untersuchungen werden an anderer Stelle im Laufe der Jahre 1987 und 1988 veröffentlicht.

Zur Zeit liegen bereits die Ergebnisse der vergleichenden Prüfung der Bruchschlagarbeit vor, die trotz ihrer bekanntermaßen großen Streuung ein sehr empfindliches Indiz für mögliche Holzschädigungen darstellt. Diesbezüglich zeigen Proben gesunder und kranker Tannen und Fichten von allen 4 Standorten keine signifikanten Unterschiede. Dies gilt auch für die Proben aus dem peripheren Stammbereich, in welchem die beschriebenen Unterschiede von Splintbreite und Wasserhaushalt festgestellt worden sind. Im übrigen steht dieser Befund im Einklang mit mehreren neuen Vergleichsuntersuchungen der Festigkeit gesunder und kranker Nadelbäume (Azmutat et al. 1986; Frühwald et al. 1984; Frühwald 1986; Grammel, Becker 1985; Schulz 1986).

Hingegen ist zu erwarten und in Einzeluntersuchungen auch bereits bestätigt worden (Graf 1987), daß die geringe Splintbreite kranker Nadelbäume vor allem bei Fichte und Kiefer zu entsprechend verringerter Tränktiefe bei der technischen Imprägnierung von waldfrischem Rundholz führt; der Kern dieser Hölzer ist bekanntlich nicht tränkbar. Dies betrifft vor allem die Imprägnierung von Masten und Palisaden. Wenn hierbei die Tränktiefe - analog zu den um 20 bis $40 \%$ bzw. um 1 bis $2 \mathrm{~cm}$ geringeren Splintbreiten - bei den kranken Fichten geringer ist, können die nach der Imprägnierung auftretenden radialen Trocknungsrisse die imprägnierte Splintzone eher überbrücken, als dies bei gesunden Bäumen mit breiterem Splint der Fall ist. Dadurch vergröBert sich die Gefahr des Befalls durch holzzerstörende Pilze über die Trocknungsrisse; eine Verringerung der Standdauer der Masten wäre die Folge. Es empfiehlt sich, diesen Gesichtspunkt bei der Auswahl des Mastenholzes zu berücksichtigen. 


\section{Literatur}

Arnold, M.: Schnell, G.R.; Sell, J. 1986: Vergleichende Splintflächcnbestimmung waldfrischer Nadelholz-Stammquerschnitte. Holz Roh-Werkstofr 44:432

Azmutat, H.; Koltzenburg, C. H.: Weiss, W.J. 1986: Untersuchungen der Holzeigenschaften von Fichte und Buche aus immissionsexponierten Beständen von Hils und Solling. Holz Roh-Werkstoff 44:301

Bauch, J. 1986: Biologische Eigenschaften des Holzes kranker Fichten aus Waldschadensgebieten zur Beurteilung der Schadenursachen und Schadwirkungen. Holz Roh-Werkstoff 44:302

Burmester, H. 1980: Holzeuchtigkeit in Nadelhölzern. Jahreszeitliche Einflüsse auf die Eigenschaften des Splint- und Kernholzes von Nadelbäumen. Holz-Zbl. 106:1303-1304

Frühwald, A. 1986: Technological properties of wood from trees in polluted regions. IAWA-Bull. 7:389-397

Frühwald, A.; Bauch, J.; Göttsche-Kühn, N. 1984: Holzeigenschaften von Fichten aus Waldschadensgebieten. Holz Roh-Werkstoff 42:441-449

Graf. E. 1987: Analyse des Einflusses des Gesundheitszustandes der Bäume auf die Imprägnierfähigkeit des Rundholzes im Wechseldruckverfahren. EMPA-Abt. Biologie, Nr. 23'1365/1 (unveröffentlicht)

Grammel, R.H.; Becker, G. 1985: Untersuchungen der Holzqualität erkrankter Fichten und Tannen wichtiger Baden-Württembergi- scher Vorkommen auf der Basis von Werkstoff- und Gebrauchsprüfung. 2. Zwischenber. an Kernforsch. Zentr. Karlsruhe, verfaßt vom Inst. Forstbenutzung, Alb. Ludwig Univ. Freiburg i. $\mathrm{Br}$

Hapla, F. 1986: Splint- und Kernanteile an Kiefern unterschiedlicher Inmissionsschadstufen. Holz Roh-Werkstoff 44:361

Huber, B. 1956: Die Gefäßleitung. In Ruhland, W.: Handbuch der Pflanzenphysiologie, Bd. IIl, p. 541-582. Berlin: Springer

Kučera, L. 1986: Kernspintomographie und elektrische Widerstandsmessung als Diagnosenmethoden der Vitalität erkrankter Bäume. Schweiz. Z. Forstwes. 137:673-690

Langner, W. 1932: Die Wasserverteilung im Stammholz der Fichte und ihre Veränderungen. Bot. Archiv 34:1-47

Rademacher, P.; Bauch. J.: Puls, J. 1986: Biological and chemical investigations of the wood from pollutions-affected pruce (Picea abies Karst.). Holzforschung 40:331-338

Schnell, G.R.; Arnold, M.; Sell, J. 1987: Wassergehalt und Splintanteil unterschiedlich vitaler Fichten und Tannen von vier schweizerischen Standorten. Forsch. u. Arbeitsber. EMPA-Abt. Holz Nr. $115 / 15$

Schulz, H. 1986: Festigkeit und Wassergehalt in Fichten, Kiefern und Buchen unterschiedlicher Schadstufen. Holz Roh-Werkstoff 44:300-301

Trendelenburg, R.; Mayer-Wegelin, H. 1955: Das Holz als Rohstoff. 2. Aufl. München: Hanser
Chin, Ch.-W.; Ampong, K.: Preliminary investigations into the treatment of messmate (Eucalyptus obliqua l'Herit.) by a modified form of double-diffusion (Vorläufige Ergebnisse von Untersuchungen zur Behandlung von Eucalyptus obliqua Masten durch ein modifiziertes Doppel-Diffusionsverfahren). Holzforschung 39 (1985) H.4: 195201; 5 Abb., 1 Tab.

Die Autoren geben einen kurzen Überblick der Literatur des herkömmlichen Doppel-Diffusionsverfahren und beschreiben anschlieBend eine neue Variante. Diese besteht aus einer Vorwärmung des untersuchten Rundholzes (Eucalyptus obliqua L'Herit.) in $100^{\circ} \mathrm{C}$ heißem Wasser für 150 Minuten und anschließendem Tauchen bei Raumtemperatur in Kupfersulfatlösung für $20 \mathrm{~h}$ und danach in eine Lösung von Arsensäure und Natriumdichromat für $48 \mathrm{~h}$. Eine Beslimmung der aufgenommenen Mengen an Kupfer, Chrom und $\mathrm{Ar}$ sen und deren Verteilung erfolgte mittels AAS. Erste Ergebnisse zeigen eine verbesserte Kupferaufnahme, die weitgehend über die Hirnenden erfolgt, wobei das Splintholz von schwächeren Dimensionen vollständig durchtränkt werden konnte. Eine Diffusion von Chrom und Arsen hingegen blieb auf die äußeren $3 \mathrm{~mm}$ begrenzt. Die sich daraus ergebenden Konsequenzen der Wirksamkeit von im DoppelDiffusionsverfahren eingebrachten Chrom-, Kupfer- und Arsenverbindungen werden diskutiert.

R.-D. Peek

Parameswaran, N.; Wilcox, W.W.; Côté, W.A.: Transmission electron microscopy and $x$-ray microanalysis of potassium permanganate staining of pentachlorophenol in treated Douglas-fir (Transmissionselektronenmikroskopie und Röntgen-Mikroanalyse der Kaliumpermanganatfärbung von Pentachlorphenol in imprägniertem Douglasienholz). Holzforschung 39 (1985) H. 5: 259-266; 19 Abb.

Wasserlösliche Holzschutzmittel führen zu einer Quellung des Holzes und ermöglichen so das Eindringen des Wirkstoffes in die Zeliwandkapillaren. Weniger bekannt ist, wie und in welchem Umfang dieser Vorgang bei der Verwendung von Lösungssystemen erfolgt, die keine Quellung des Holzes bewirken, wie z. B. Pentachlor- phenol in verflüssigtem Petroleumgas oder Methylchlorid. In Fortführung früherer Arbeiten wurde eine Methode für die Transmissionselektronenmikroskopie (TEM) zur Kontrastierung von Pentachlorphenol mit Kaliumpermanganat angewendet und mit den $\mathrm{E}_{\mathrm{r}}$ gebnissen von Röntgen-Mikroanalysen (REM-EDXA) verglichen Im TEM zeigte PCP-imprägniertes Douglasienholz in den Tracheidenwänden dunkel kontrastierte "Kristalle“, die REM-EDXA-Analyse bestätigte das gleichmäßige Vorkommen von PCP in den Zellwänden. Zufriedenstellende Kontrastierungen von TEM-Proben wurden nur nach mehrmonatiger Lagerung des Holzes in FEA (Formalin/Essig/Alkohol) erzielt. Offensichtlich wird das PCP durch den Alkohol mobil, so daß es zur Bildung von „Kristallen" kommen kann.

M. Rütze

Panow, S.; Nester, M. R.: Drying end point determination during hightemperature drying (Bestimmung des Trocknungszeitendes während der Hochtemperaturtrocknung). Forest Prod. J. 35 (1985) No. 10: $51-55 ; 4 \mathrm{Abb} ., 5 \mathrm{Tab}$.

Ein Problem bei der Hochtemperatur-Kammertrocknung ist die Entscheidung, wann das Trockengut den gewünschten Feuchtigkeitsgehalt erreicht hat. Eine brauchbare Methode zur Bestimmung der Beendigung einer Trocknung liefert das Verhältnis zwischen der Holzfeuchtigkeit und der Holztemperatur. Dies wurde an acht Versuchstrocknungen (sechs davon in einem Labor- und zwei in einem industriellen Trockner) mit slash pine (Pinus elliottii Engelmann var. elliottii) nachgewiesen. Bei den Trocknungen wurde die Temperatur mit Hilfe von Kupfer-Konstantan-Thermoelementen, die durch 3,5 mm-Bohrungen in den Kern von Probebrettchen eingeführt wurden, überwacht. Wenn $80 \%$ der Probebrettchen die Temperatur von $100^{\circ} \mathrm{C}$ überschritten, wurde die Trocknung beendet. Die Versuchsergebnisse sind in mehreren Tabellen zusanmengestellt. Die Temperaturanzeige der Thermoelemente läßt sich für die automatische Bestimmung der Trocknungsdauer bei Erreichen der gewünschten Holzfeuchtigkeit anwenden. 